\title{
PERFORMANCE CHARACTERISTICS OF A HYBRID WING FOR UAV
}

\author{
Aravind K.S $\mathbf{S}^{1}$, Naveena K.P \\ ${ }^{1,2}$ Project Assistant, Department of Mechanical Engineering, PES Institute of Technology, Bangalore-560085, India; \\ aravind4391@gmail.com,naveeng602@gmail.com
}

\begin{abstract}
Unmanned aerial vehicles (UAV) have become important in the surveillance sector of various governments. Their adavatage of high end technology and minimal risk of human life has been the major boost with respect to the research associated with UAVs. Over the years, the designing and optimization of the aerodynamics of these vehicles has gained lot of importance owing to the increasing urge to develop UAVs with better stability and endurance at subsonic speeds. Since the aircraft should have various performance characteristics as per the desired aerodyanamic requirements, it has been a challenging task to incorporate these characteristics through the use of a single homogeneous airfoil for the wing. With regard to the above discussion, this work deals with the comparison of performance characteristics of a hybrid wing consiting of two airfoil sections combined into a single wing and those of wings with each of the two homogeneous airfoil sections. The optimal wing dimensions for the surveilence UAV was obtained through MATLAB. Three separate models consiting of the hybrid wing, S1223 and E420 were developed through Solid Edge and imported into Fluent. Later, the wing models were subjected to aerodynamic analysis through the implementation of parameters experienced by the wing during the actual flight. The results obtained were compared in order to draw a conclusion regarding the advantages of using a hybrid wing as against the usage of wings with homogeneous airfoil.
\end{abstract}

Keywords: UAV, Hybrid wing, Eppler 420, Seilig 1223 and Fluent. $* * *$

\section{INTRODUCTION}

In the recent years the advantages of using UAVs has increased exponentially for defence surveillance and wild life exploration. Its ability to provide the required data without the risk of human life with an added value of being able to explore regions which are infeasible for humans. These advantages resulted in a vast potential yet to be tapped. Since the research on UAVs is still in primitive stages as compared to the research on manned aircrafts, a lot of minor issues are still present which provides the scope for further research and development in the field of UAVs. These aircrafts are built to sustain long hours of flight in varying conditions of atmosphere. Owing to this challenge which needs to be countered, research on the aerodynamics of the UAV's wing is given major attention among the various research domains assosiated with UAVs.

Hybrid wings is a concept that has been in the minds of researchers from quite some time, these wings are known to be successful in implementing aerodynamic characteristics of multiple airfoils[1]. Considering the problem in hand which demands for an UAV capable of having high lift in order to save power and high stability in order to improvise the manuvering control over the aircraft during the turbulent conditions, implementaion of Hybrid wings is a feasible option in overcoming the problem mentioned above.
Two airfoils namely Eppler 420 and Selieg 1223 were considered as primary profiles of the hybrid wing. E420 has a thicker cross section which accounts for the sturdiness of the wing and results in better stability during wind gusts. On the other hand the thin cross section of S1223 aids in higher lift and lesser stability as compared to E420[2].

In the light of the above discussion, the hybrid wing consiting of E420 at root and S1223 at tip is modelled and analysed in Fluent in order to obtain its perforamnce characteristcs with the usage of CFD tool.

\section{METHODOLOGY}

The initial design began with the assumed values obtained from the references and from the 'Available Standard Models', based on the desired requirements. The desired requirements consisted of a cruise speed of $10 \mathrm{~m} / \mathrm{s}$ and stall velocity of $5 \mathrm{~m} / \mathrm{s}$ for a gross weight of $1.2 \mathrm{~kg}$ to $1.4 \mathrm{~kg}$ (including the payload requirement of $0.3 \mathrm{~kg}$ to $0.4 \mathrm{~kg}$ ).

The first major step refers to the determination of the wing loading parameters. The fundamental laws were applied to the system to obtain the suitable equations for landing distance, stall velocity, cruise speed. As a result the fundamental laws were brought down to three sets of equations which were programmed suitably in the MATLAB to know the various configurations possible. 
During the iterative process, various data were assumed. It involves maximum landing distance of $15 \mathrm{~m}$ and maximum take off distance of $20 \mathrm{~m}$. The airfoil parameters were the average of two different airfoils S1223 and the E420. Finally the equations were iterated for aspect ratio (AR) approx equal to 7 .

\begin{tabular}{|c|c|c|c|c|c|c|c|}
\hline $\mathrm{Cl}_{\max }$ & \multicolumn{2}{|c|}{ Votall } & Veruise & \multicolumn{2}{|l|}{ AR } & \multicolumn{2}{|l|}{$w_{0}$} \\
\hline 2.45 & \multicolumn{2}{|c|}{5.00} & 9.00 & \multicolumn{2}{|c|}{7.00} & \multicolumn{2}{|l|}{1.40} \\
\hline $\mathrm{WS}_{\min }$ & WSpos & $\mathrm{V}_{\text {stall }}$ & $\mathrm{TW}$ & $>=$ PowR & $\mathrm{AR}$ & $S_{r e f}$ & $<=$ climbGrad \\
\hline \multirow[t]{3}{*}{32.90} & 37.53 & 5.00 & 0.26 & 17.32 & 7.64 & 0.37 & 0.18 \\
\hline & 48.36 & 5.68 & 0.56 & 42.12 & 7.64 & 0.28 & 0.48 \\
\hline & 32.90 & 4.68 & 0.19 & 11.93 & 7.64 & 0.42 & 0.11 \\
\hline pan & Root & Tip & $\begin{array}{l}\text { Sref }\end{array}$ & \multicolumn{2}{|c|}{$\mathrm{AR}$} & & \\
\hline .40 & 0.21 & 0.20 & 0.28 & \multicolumn{2}{|l|}{6.90} & & \\
\hline .40 & 0.22 & 0.19 & 0.28 & \multicolumn{2}{|l|}{6.90} & & \\
\hline .40 & 0.23 & 0.18 & 0.28 & \multicolumn{2}{|l|}{6.90} & & \\
\hline .40 & 0.24 & 0.17 & 0.28 & \multicolumn{2}{|l|}{6.90} & & \\
\hline .40 & 0.25 & 0.16 & 0.28 & \multicolumn{2}{|l|}{6.90} & & \\
\hline .60 & 0.23 & 0.23 & 0.37 & \multicolumn{2}{|l|}{7.00} & & \\
\hline .60 & 0.24 & 0.22 & 0.37 & \multicolumn{2}{|l|}{7.00} & & \\
\hline .60 & 0.25 & 0.21 & 0.37 & \multicolumn{2}{|c|}{7.00} & & \\
\hline & $-----R$ & ectang & lar wir & \multicolumn{2}{|c|}{ Configuration- } & ---- & \\
\hline pan & Root & Tip & Sref & \multicolumn{2}{|c|}{$\mathrm{AR}$} & & \\
\hline .00 & 0.28 & 0.28 & 0.28 & \multicolumn{2}{|l|}{7.04} & & \\
\hline .20 & 0.35 & 0.35 & 0.42 & \multicolumn{2}{|l|}{6.90} & & \\
\hline
\end{tabular}

Fig -1: Iterations carried out in MATLAB

Theoretically the smaller is the wing loading (WS) the better is the gliding and other flight performance parameters at the cost of increasing gross weight, as a result a suitable compromise has to be made between the wing loading and the gross weight. Accordingly the WS was chosen to be 48.36 . The red colored numbers shows the desired parameters for this wing loading. Consequently, the plane can have a gross weight of $1.3 \mathrm{~kg}$ which is in the close proximity to the desired gross weight of $1.4 \mathrm{~kg}$. On the other hand the Aspect Ratio (AR) $=6.9$ is also found to be in the expected range $(\sim 7)$.

Theoretically tapered wings are preferred as compared to rectangular wing. One major reason is to get Elliptical Lift Distribution on the wing and to have high aspect ratio for the same reference area and same wing material.

In order to avoid the Tip stall the airfoils of high Lift Coefficients have to be placed at the tip region. In our case S1223 is placed at the tip. This has an added advantage with respect to the structural behavior. The Eppler (E420) is thicker than Seleig (S1223), as a result the wing can be structurally sturdy in the root region and also it provides sufficient empty space to keep the payload (if required) in the wing.

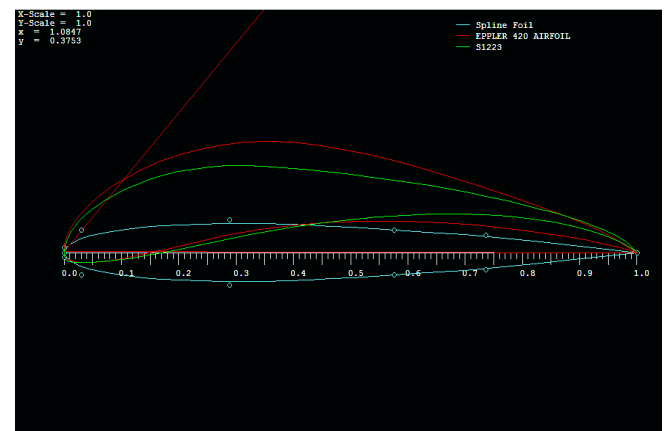

Fig -2: Depicting the relative thickness of the two airfoils that are superimposed.

The modelling of the wing was done in Solid Edge with the help of a tool 'Lofted protrusion'. The E420 cross section was sketched on a vertical plane and the S1223 was sketched on a parallel plane at a distance equal to half the wing span. 'Lofted protrusion' was used to generate a solid model starting from E420 at the root and S1223 at the tip. The intermediate airfoil shapes were obtained by taking cross sections of the wing at various distances from the root.

The next stage was to determine the accurate aerodynamic parameters from the CFD techniques through Fluent software.
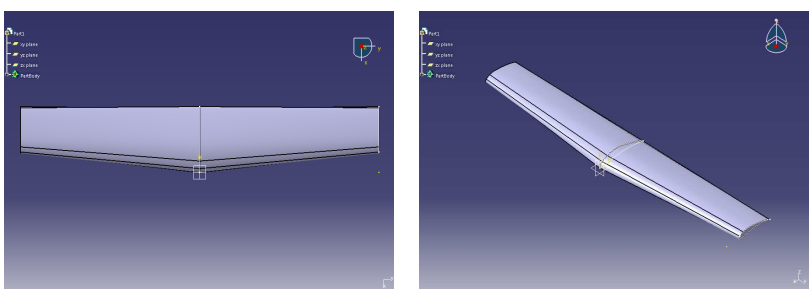

Fig -3: Solid models of Hybrid wing

In order to check the 2D grid independence, the flow analysis was done for NACA4412 airfoil for which the experimental values are available in the references. Later it was extended to $3 \mathrm{D}$ wing model to get the realistic view and to solve the problems related to downwash. It was found that an error of $10 \%$ to $11 \%$ exists in the analysis which is reasonable and is in accordance with the CFD techniques [3]. Conventionally the wing will be made of the same airfoil sections due the lack of computational/experimental results for varying airfoil sections. Since we had CFD techniques at our disposal we thought of using the varying cross section and varying airfoil type for our wing purely as a state of art. In order to check the relative performance of this Hybrid wing made of S1223 and E420, independent analysis was carried on each of them. Theoretically the hybrid wing characteristics should fall between the characteristics of two pure wings. Accordingly the results obtained matched with the predicted values. 


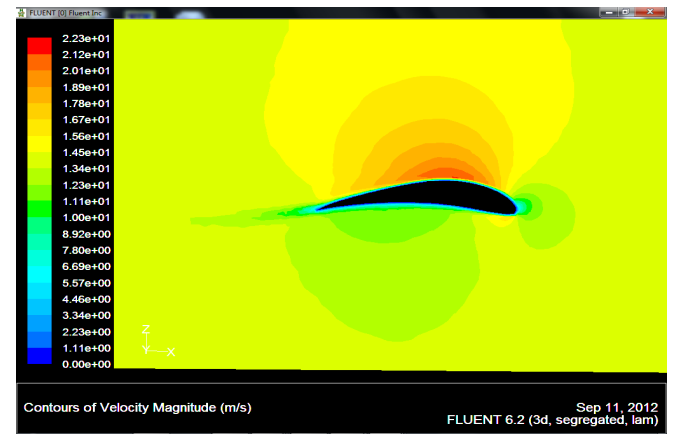

Fig -4: Velocity contours.

After the iterations, co-efficient of lift, drag and moment were obtained from Fluent. These iterations were repeated for various angles of attack and also with three models namely pure E420, pure S1223 and hybrid wing. The resulting data was plotted in order to verify has to whether the characteristics of a hybrid wing lies in between that of pure E420 and pure S1223.

In order to obtain the lift distribution curve a complicated technique was used. Once the iterations were over cross sections of the wing taken at regular intervals from the root in order to obtain the airfoil flow data at those sections

At these sections the nodes which were very close to the outer surface of the airfoil were considered and the pressure at these nodes were calculated and tabulated. These pressure values were plotted along the chord of the airfoil in order to obtain the pressure distribution curve. The area enclosed by the pressure distribution curve was calculated through integration in MATLAB which resulted in obtaining the lift generated by airfoil at that section. The procedure was repeated for all the sections including that of root and tip.

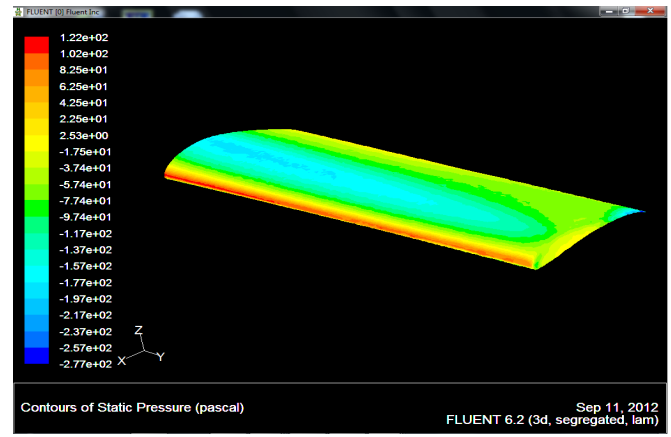

Fig -5: Pressure contours.

\section{RESULTS AND DISCUSSIONS}

The analysis was conducted to come into a conclusion regarding two aspects which are 'lift distribution curve' and plots of $\mathrm{Cm}, \mathrm{Cl}$ and $\mathrm{Cd}$ versus angle of attack [4].

\subsection{Lift Distribution Curve}

The ideal lift distribution curve for a wing is elliptical where the maximum is at the root and reaches zero at the tip. However, the lift distribution curve obtained for the hybrid wing had a $\mathrm{Cl}$ value varying between 1.4 and 1 until $85 \%$ of the half wing span. This result helps in drawing a conclusion that the lift distribution curve of this hybrid wing has a better consequence on the flight parameters of the aircraft. It can be associated with the $\mathrm{Cl}$ falling into a narrow band for more than $85 \%$ which means that the lift over the span of the wing is more or less a constant value which aids in better endurance of the aircraft and a stable flying condition during wind gusts as compared to elliptic lift distribution curve.

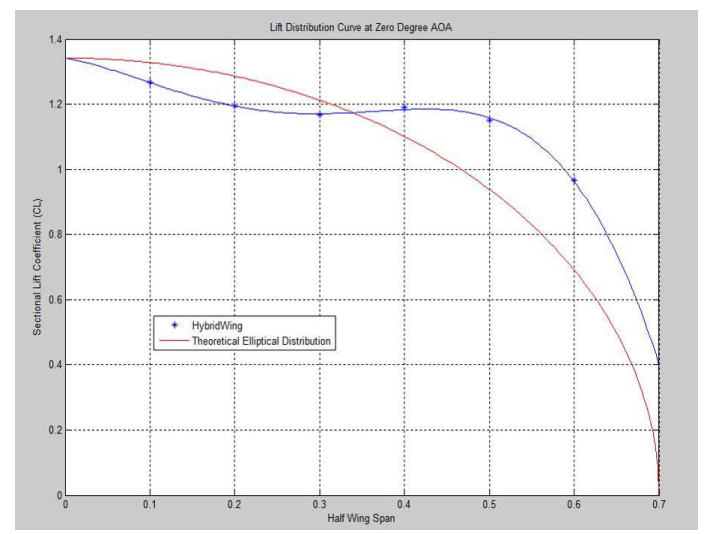

Fig -6: Lift distribution curve.

\subsection{Cm, Cl \& Cd vs AOA}

The co-efficients of drag, lift and moment were obtained from the software and these values were plotted against the iterations executed for angles of attack starting from zero degree with an increment of two degrees [4, 5]. It can be seen that the Hybrid profile matches suitably with the intermediated values. One prominent point that is to be inferred from the plots is that the Seleig although has greater lift coefficient $(\mathrm{Cl})$, it causes greater drag coefficient $(\mathrm{Cd})$ in the working range of Reynolds number of 2lakhs.

The co-efficients of the hybrid wing was found to lie in between that of pure E420 and pure S1223. These plots verified the prediction that the hybrid wing has the performance characteristics that lies in between those of its parent airfoils. 


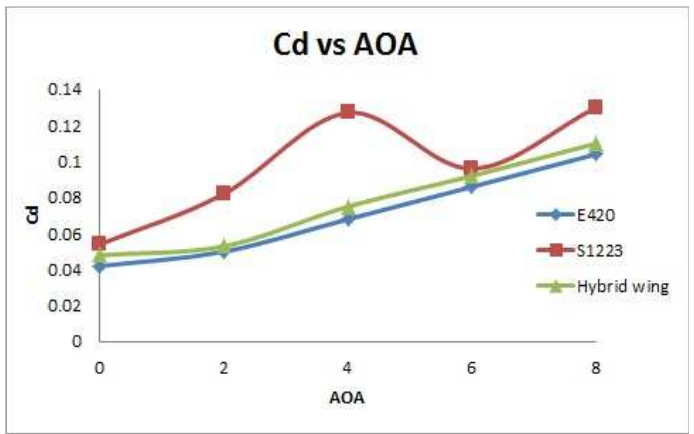

Fig -7: Graph of Cd vs AOA for three different airfoils.

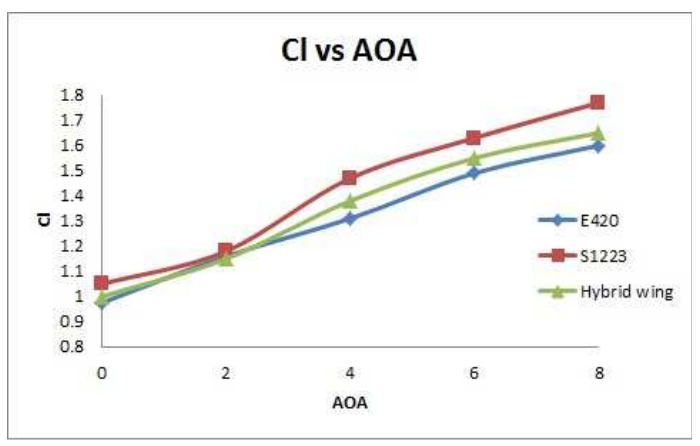

Fig -8: Graph of Cl vs AOA for three different airfoils.

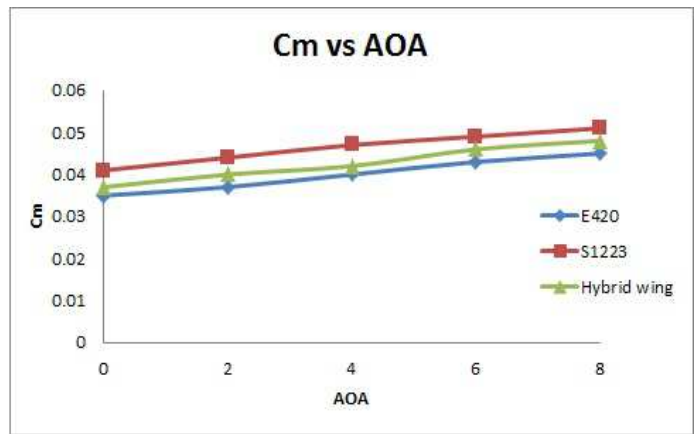

Fig -9: Graph of Cm vs AOA for three different airfoils.

\section{CONCLUSIONS}

The inferences drawn from this work is two-fold in nature. Firstly, the performance characteristics namely the coefficients of lift, drag and moment lie within the predicted region as per the CFD verification. Secondly, the lift distribution curve along the wing span has shown a desired plot which accounts for the characteristics suitable for a UAV with high endurance.

However, certain aspects of the analysis have a scope for improvement. The modelling of the hybrid wing was done using a homogenous interpolation technique where the intermediate airfoils of the solid model cannot be tweaked as per the requirements. The intermediate airfoils can sometimes be neither that of E420 nor S1223. A major change that can be implemented is that the wing can be divided into two section where one section comprises of S1223 while the other comprises of E420, these sections' proportions can be varied to get the desired results and also the transition from one airfoil to another can be made smooth there by successfully correcting the problem of intermediate airfoils having undefined cross-sections.

\section{ACKNOWLEDGEMENTS}

The authors would like to thank Prof D.Jawahar, C.E.O P.E.S Group of Institutions for his encouragement. They would also like to thank Dr K.N.B Murthy, Principal \& director, P.E.S Institute of Technology for his constant support.

\section{REFERENCES}

[1]. L. Popelka, M. Matejka, J. Nozicka, V. Uruba. "Multicriteria design of sailplane airfoils multicriteria design of sailplane airfoils", ISTP-16, 2005, PRAGUE 16TH international symposium on transport phenomena.

[2]. Aniket C. Aranake, Vinod K. Lakshminarayan, Karthik Duraisamy; "Computational Analysis of Shrouded Wind Turbine Configurations"; 51st AIAA Aerospace Sciences Meeting including the New Horizons Forum and Aerospace Exposition 07 - 10 January 2013, Grapevine (Dallas/Ft. Worth Region), Texas.

[3]. Anastasios P. Kovanis, Vangelis Skaperdas, John A. Ekaterinari; "Design and analysis of a light cargo uav prototype"; 4th ANSA \& $\mu$ ETA International Conference.

[4]. T. F. G. Costa, E. M. Belo; "Prediction of aerodynamics characteristics and airloads of a generic geometry wing"; 25th international congress of the aeronautical sciences.

[5]. David L. Rodriguez, Peter Sturdza, Yoshifumi Suzuki, Hervé Martins-rivas; "A Rapid, Robust, and Accurate Coupled Boundary-Layer Method for Cart3D"; Desktop Aeronautics, Inc., Palo Alto, CA, 94303.

\section{BIOGRAPHIES}

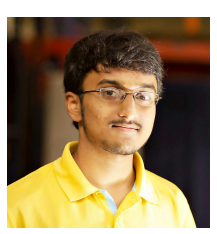

Aravind $\mathrm{K} \mathrm{S}$ received his $\mathrm{BE}$ degree in Mechanical Engineering from PES Institute of Technology, Bengaluru in 2013. Presently, he is working as Project Assistant in PES Institute of Technology since May, 2013. He has published 1 international paper. And he has also obtained NASA certification for the participation in an international event. 
Naveena K P received his BE degree in Mechanical Engineering from PES Institute of Technology, Bengaluru in 2013. Presently, he is working as Project Assistant in PES Institute of Technology. He has published 3 international papers. 